\title{
PCSK9 in Haemostasis and Thrombosis: Possible Pleiotropic Effects of PCSK9 Inhibitors in Cardiovascular Prevention
}

\author{
Francesco Paciullo ${ }^{1}$ Stefania Momi ${ }^{1} \quad$ Paolo Gresele $^{1}$ \\ ${ }^{1}$ Division of Internal and Cardiovascular Medicine, Department of \\ Medicine, University of Perugia, Perugia, Italy \\ Thromb Haemost 2019;119:359-367. \\ Address for correspondence Paolo Gresele, MD, PhD, Division of \\ Internal and Cardiovascular Medicine, Department of Medicine, \\ University of Perugia, Strada Vicinale Via delle Corse, 06126 Perugia, \\ Italy (e-mail: paolo.gresele@unipg.it).
}

\begin{abstract}
Keywords

- atherothrombosis

- CD36

- dyslipidaemia

- FVIII

- LOX-1

Since increased cholesterol levels are crucial in determining the development of atheroma, their reduction represents a mainstay in primary and secondary cardiovascular prevention. The most recent spectacular advancement in cholesterol-lowering therapy is represented by proprotein convertase subtilisin/kexin type-9 (PCSK9) inhibitors. Although their benefit over currently available treatments has been ascribed primarily to their strong low-density lipoprotein (LDL)-cholesterol reducing action, several clues suggest that PCSK9 inhibitors may also influence platelet function and blood coagulation. PCSK9 knockout mice develop less venous and arterial thrombosis and show reduced in vivo platelet activation upon arterial injury. In patients with acute coronary syndromes (ACSs) treated with $\mathrm{P}_{2} \mathrm{Y}_{12}$ inhibitors, a direct association between PCSK9 serum levels and residual platelet reactivity was found. A direct correlation between urinary excretion of 11-dehydro-thromboxane- $B_{2}$, a marker of in vivo platelet activation, and circulating PCSK9 levels was reported in patients with atrial fibrillation. Moreover, recombinant human PCSK9 added in vitro to human platelets potentiated activation induced by weak agonists. Finally, blood clotting factor VIII (FVIII), which is associated with stroke and ACS risk, is cleared from the circulation by members of the LDL receptor (LDLR) family. Given that PCSK9 degrades LDLR, it is conceivable that PCSK9 inhibitors by enhancing the expression of LDLR may slightly decrease circulating FVIII, in this way contributing to the prevention of cardiovascular events. This review aims to discuss the possible and hypothetical interactions between PCSK9 and the haemostatic system and to examine the possible pleiotropic effects of PCSK9 inhibitors in cardiovascular prevention.
\end{abstract}

\section{PCSK9, Lipid Metabolism and Cardiovascular Risk}

Cholesterol is transported in the bloodstream by lipoprotein particles. The two major cholesterol-carrying lipoproteins are low-density lipoproteins (LDLs) and high-density lipoproteins (HDLs). LDLs, whose main protein fraction is apolipoprotein B100, transport cholesterol from the liver to peripheral tissues, including the arterial walls. ${ }^{1}$ LDLs are a major determinant of atherosclerosis, and both American and European guidelines recommend specific LDL threshold reductions to prevent cardiovascular events. ${ }^{2}$ On the other hand, HDLs, whose main protein fraction is apolipoprotein AI, transport cholesterol from peripheral tissues to the liver, facilitating its clearance. ${ }^{3}$ Circulating cholesterol levels are regulated by the balance between their biosynthesis and received

March 19, 2018

accepted after revision

November 19, 2018 (c) 2019 Georg Thieme Verlag KG Stuttgart · New York
DOI https://doi.org/ 10.1055/s-0038-1676863. ISSN 0340-6245. 
clearance. The rate-limiting step in cholesterol biosynthesis is the conversion of 3-hydroxy-3-methyl glutaryl coenzyme A (HMG-CoA) into mevalonate catalyzed by HMG-CoA reductase, which represents the pharmacologic target of statins, the most widely used cholesterol-lowering agents. ${ }^{4}$ LDL clearance takes place via specific LDL receptors (LDLRs) in the liver which bind apolipoprotein B100 forming a complex which is internalized. ${ }^{5}$ The LDL component of the complex is then degraded by lysosomal enzymes, while LDLR are recycled to the cell membrane to bind other LDL particles. ${ }^{6}$ The expression of LDLR on the hepatocyte surface is regulated by intracellular cholesterol levels, with lower intracellular cholesterol leading to increased surface LDLR, and vice versa. ${ }^{7}$ LDLRs are also regulated by proprotein convertase subtilisin/kexin type-9 (PCSK9), originally called Narc-1, an enzyme which fosters their degradation. PCSK9 is mainly synthesized by the liver, but also by the brain, kidney, intestine, pancreas and steroidogenic tissue, and is found both in the intra- and extracellular space. Extracellularly, PCSK9 binds the first epidermal growth factor-like repeat of LDLR forming a tri-molecular complex (LDLR-LDL-PCSK9) which is then internalized. Once inside the hepatocyte, PCSK9 prevents LDLR from escaping lysosomal degradation, and therefore to recycle to the cell surface, thus reducing their expression. Intracellularly, PCSK9 binds nascent LDLR and targets them to lysosomes where they are degraded. ${ }^{8}$ The interest in PCSK9 has sharply risen after the observation that gain of function variants of its gene are associated with enhanced levels of circulating LDL cholesterol (LDL-c), and thus with increased cardiovascular risk. ${ }^{9}$ Conversely, carriers of loss-of-function variants have low LDL-c and a reduced risk of ischaemic cardiovascular disease. ${ }^{10}$ Moreover, in a seminal study, the adenoviral-mediated over-expression of PCSK9 in mice caused a striking increase in plasma cholesterol. ${ }^{11}$ Since the discovery of the important role of PCSK9 in lipid metabolism, numerous approaches to its inhibition have been attempted, leading to the development of monoclonal antibodies (MoAbs) blocking its extracellular action, probably the most relevant for the regulation of circulating cholesterol levels. ${ }^{12}$ Several phase III trials with anti-PCSK9 MoAb have been performed in patients with hypercholesterolemia and high cardiovascular risk and, based on their positive results in terms of safety and efficacy, two fully humanized anti-PCSK9 MoAbs, alirocumab and evolocumab, have received Food and Drug Administration (FDA) and European Medicines Agency approval in 2015 as a second line treatment, in addition to diet and maximally tolerated statin therapy, to lower LDL-c in adults with heterozygous familial hypercholesterolaemia or clinical atherosclerotic cardiovascular disease who require additional LDL-c lowering. Recently, based on the results of the landmark FOURIER trial, ${ }^{13}$ the FDA has approved evolocumab also to prevent heart attacks, strokes and coronary revascularization in adults with established cardiovascular disease. In this trial, including more than 27,000 high-risk patients with atherosclerotic cardiovascular disease with a LDL-c of $\geq 70 \mathrm{mg} / \mathrm{dL}$ who were receiving statin therapy, subjects randomized to evolocumab showed a significantly lower incidence of major adverse cardiovascular events compared with those randomized to placebo. ${ }^{13}$ Moreover, a meta-analysis of 24 phase II and phase III trials involving 10,159 adults with hypercholesterolaemia, on statin treatment or not, has shown that anti-PCSK9 MoAbs significantly reduce all-cause mortality compared with no anti-PCSK9 treatment (odds ratio [OR], 0.45 [95\% confidence interval $\mathrm{Cl}, 0.23-0.86], p=0.015) .^{14}$ Thus, PCSK9 inhibitors appear to be a very effective strategy to prevent cardiovascular events.

\section{Pleiotropic Effects of Lipid-Lowering Interventions}

Since the detrimental effect of LDL-c on the cardiovascular system is concentration-dependent, the reduction of ischaemic cardiovascular events produced by PCSK9 inhibitors when added to statins has been ascribed primarily to their further, strong LDL-c-lowering action. ${ }^{15}$

However, statins may exert cardiovascular protective actions independent from LDL-c lowering which have been called pleiotropic effects. ${ }^{16}$ The reduction of cardiovascular events attained with statins in some clinical trials, like the JUPITER trial, ${ }^{17}$ has been indeed greater than that expected solely from LDL-c reduction. ${ }^{18,19}$ In fact, cholesterol-independent beneficial effects of statins on the cardiovascular system, such as the stabilization of atherosclerotic plaques, reversal of endothelial dysfunction, blunting of inflammation, enhancement of fibrinolysis and inhibition of platelet activation and blood coagulation, have been well documented. ${ }^{20-23}$

On the other hand, in a recent meta-analysis of trials with lipid-lowering interventions including more than 300,000 patients, the relative risk reduction of major vascular events associated with PCSK9 inhibitors use was higher, even if not significantly, than that observed with statins for the same LDL-c reduction (OR, 0.49 [95\% CI, 0.34-0.71] vs. 0.61 [95\% CI, $0.58-0.65]) .{ }^{24}$ Reasons advocated for this observation are the raising activity on anti-atherogenic HDL and the ability to reduce lipoprotein (a) $[\operatorname{Lp}(a)]$ of PCSK9 inhibitors. ${ }^{25,26}$ However, pleiotropic effects of PCSK9 inhibitors independent from lipid metabolism can also be considered. ${ }^{27}$

Indeed, many pre-clinical and clinical data support the hypothesis that the cardiovascular protective effect of PCSK9 inhibitors may be more complex, involving mechanisms which go beyond their lipid-lowering action, several of which may affect the haemostatic system.

\section{Hints for Pleiotropic Effects of PCSK9 on Haemostasis and Thrombosis}

Haemostasis begins at a vascular injury site with platelet adhesion and aggregation (primary haemostasis), followed by the activation cascade of clotting factors (secondary haemostasis). Thrombosis, which can be considered an excessive extension of a haemostatic reaction, occurs in the arterial and venous vascular beds by mechanisms which differ in relation to the anatomical and rheological characteristics of these two systems. In arteries, thrombosis originates from the rupture of atherosclerotic lesions and is mainly generated by platelet 
activation, ${ }^{28}$ while in veins, thrombi are mainly the consequence of clotting activation favoured by stasis, hypercoagulability and endothelial damage. ${ }^{29}$ However, several recent observations suggest that these two pathways contribute to both arterial and venous thrombosis. In fact, risk factors for ischaemic cardiovascular disease, among which hyperlipidaemia, induce endothelial dysfunction in both the arterial and venous vascular beds, leading to the development of either arterial or venous thrombosis depending on the concomitant inciting conditions..$^{30,31}$

\section{Studies in Animals}

The discovery of the central role played by PCSK9 in lipid metabolism has also been grounded on studies performed in gene-modified mice. The adenovirus-induced over-expression of PCSK9 in mice resulted in decreased hepatic LDLR expression with associated hypercholesterolaemia, whereas the deletion of the PCSK9 gene increased hepatic LDLR expression and reduced LDL-c circulation, ${ }^{11}$ recapitulating the phenotypes of gain- or loss-of-function PCSK9 variants in humans.

Interestingly, $\mathrm{PCSK}^{-1-}$ mice showed a reduction of $\mathrm{FeCl}_{3}$ injury-induced carotid artery thrombosis, with the formation of unstable non-occlusive thrombi, ${ }^{32}$ suggesting an impaired platelet function. Indeed, in these mice the activation of circulating platelets provoked by arterial injury, shown by increased glycoprotein (GP) IIb/IIla activation, Pselectin expression and circulating platelet-leukocyte aggregates, was strikingly reduced as compared with control mice. ${ }^{32}$ It cannot be disregarded, however, that the assessment of in vivo platelet activation in mice by the measurement of these biomarkers may be prone to errors. ${ }^{33}$

Moreover, the hypercoagulable state induced by sepsis was exacerbated in PCSK9 over-expressing mice, as shown by enhanced thrombin-antithrombin complexes and reduced protein C plasma levels, ${ }^{34}$ suggesting that changes in PCSK9 may also have an impact on blood coagulation.

Indeed, PCSK9 ${ }^{-1-}$ mice developed significantly smaller venous thrombi as compared with wild-type mice after inferior vena cava ligation. ${ }^{35}$ Plasma factor VIII (FVIII) levels are known to modulate venous thrombosis in mice, ${ }^{36,37}$ and there are theoretical reasons to suppose that PCSK9 may modulate circulating FVIII levels (see later); thus, although no blood clotting factor measurements were made in this study, ${ }^{35}$ it is conceivable that an effect on FVIII may have contributed to reduce venous thrombosis. On the other hand, plasma levels of soluble P-selectin (sP-selectin), a platelet and endothelial activation biomarker, ${ }^{38}$ were significantly lower in PCSK9-deficient than in control mice after the induction of vena cava thrombosis, ${ }^{35}$ further suggesting that PCSK9 ${ }^{-1-}$ mice have impaired platelet function, but also that PCSK9 deletion may reduce endothelial activation. In this regard, it is interesting that increased circulating sPselectin was observed in humans with unprovoked deep vein thrombosis who have endothelial dysfunction. ${ }^{30,31}$

\section{Studies in Humans}

The possible role of PCSK9 in modulating platelet function has been assessed also in humans. In a prospective, observational study in patients with a recent acute coronary syndrome (ACS) undergoing percutaneous coronary intervention and receiving P2Y $_{12}$ inhibitors, the PCSK9-REACT study, a direct correlation between PCSK9 plasma levels and high-on-treatment platelet reactivity was observed, suggesting that PCSK9 enhances platelet activation..$^{39}$ Indeed, recent observations have shown that human recombinant PCSK9 pre-incubated in vitro with platelets potentiates aggregation, P-selectin expression and GPIIb/IIIa activation induced by a weak agonist, ${ }^{32}$ acting therefore as a primer of platelet activation. ${ }^{40}$

In this regard, it is interesting that twice as much PCSK9 was found to be contained in platelets from type 2 diabetes mellitus (T2DM) patients with coronary artery disease (CAD) than in platelets from healthy controls, ${ }^{41}$ suggesting that PCSK9 may be released during platelet activation and contribute to the well-established T2DM-associated platelet hyper-reactivity and impaired responsiveness to anti-platelet agents. ${ }^{42,43}$

Human megakaryocytes express messenger ribonucleic acid (mRNA) for PCSK9, although at low levels (A.S. Weyrich and R.A. Campbell, University of Utah, personal communication), but this is not found in platelets. ${ }^{44}$ It can therefore be hypothesized that mRNA for PCSK9 is handled by megakaryocytes similarly to mRNA for matrix metalloproteinase-2, with mRNA not sorted into platelets but the protein present, ${ }^{45}$ even if only in a platelet sub-population, ${ }^{41}$ as already reported for tissue factor $(\mathrm{TF})^{46}$ and endothelial nitric oxide synthase. ${ }^{47}$

It was found that PCSK9 plasma levels positively correlate with the platelet count and plateletcrit in patients with stable CAD, further suggesting a link between PCSK9 and platelets in patients with coronary disease. ${ }^{48}$ Furthermore, PCSK9 plasma levels have been recently reported to increase during an acute coronary event, ${ }^{49}$ a condition associated with a striking bout of in vivo platelet activation, ${ }^{50,51}$ and to positively correlate with the severity of coronary artery lesions evaluated by the SYNTAX score. ${ }^{49}$ On the other hand, platelet reactivity in ACS correlates positively with the SYNTAX score, ${ }^{52}$ further suggesting a role of enhanced circulating PCSK9 in platelet hyper-reactivity. Moreover, activated platelets of patients with CAD release soluble sortilin $^{53}$ which is known to facilitate PCSK9 secretion, ${ }^{54}$ making even more plausible the hypothesis of a positive feedback activation of platelets through PCSK9 during ACS.

A direct relationship between in vivo platelet activation and PCSK9 plasma levels has been reported also in atrial fibrillation. In this study, circulating PCSK9 correlated with urinary 11-dehydro- thromboxane $B_{2}$ excretion, ${ }^{55}$ an unbiased marker of in vivo platelet activation. ${ }^{56}$

Several clues suggest that PCSK9 may also influence secondary haemostasis in humans. In fact, plasma levels of $\mathrm{TF}$, a pro-coagulant glycoprotein triggering thrombin formation and playing a central role in atherothrombosis, ${ }^{57}$ positively correlated with plasma PCSK9 in patients with CAD and T2DM. ${ }^{58}$ Moreover, single nucleotide polymorphisms of the PCSK9 gene were shown to be associated with the development of thrombosis in carriers of anti-phospholipid antibodies, ${ }^{59}$ subjects characterized by a hypercoagulabe state and in vivo platelet activation, ${ }^{60,61}$ confirming a link between PCSK9, platelets and blood coagulation. 
Altogether, these data represent a strong hint that the association of PCSK9 with cardiovascular risk involves mechanisms that go beyond the mere regulation of circulating LDL-c and which include effects on the haemostatic system.

\section{Modulation of Platelet Activation by Lipoproteins and the Possible Influence of PCSK9 Inhibitors}

Dyslipidaemia may influence platelet reactivity and haemostasis by several mechanisms. Enhanced oxidative stress associated with high circulating LDL-c leads to the formation of oxidized-LDL (ox-LDL), which strongly contribute to inflammation-driven thrombosis ${ }^{62}$ by activating CD36 and LOX-1 receptors on platelets ${ }^{63,64}$ (-Fig. 1). CD36 is a member of the SRB-1 family which binds native- and ox-LDL and plays a role in thrombus formation, ${ }^{65}$ while LOX-1 (also called oLR-1) is a multi-ligand scavenger receptor whose expression is triggered by pro-inflammatory stimuli. ${ }^{66,67}$ In addition, activated platelets themselves oxidize LDL generating ox-LDL, in this way propagating platelet activation. ${ }^{68}$ Another mechanism by which dyslipidaemia may affect platelet reactivity is through the generation of lipid peroxide-modified phospholipids, which activate platelets by acting on Toll-like receptor $2 .{ }^{69}$
Oxidized phospholipids are transported by $\operatorname{Lp}(\mathrm{a}),{ }^{70}$ and the latter may also directly activate platelets by not yet unravelled mechanisms. $^{71}$

Finally, HDL attenuate platelet function by interacting with the ApoER2 and SRBI receptors, and also promoting cholesterol efflux from platelet membranes. ${ }^{72}$ Indeed, cholesterol incorporation in plasma membranes induces platelet hypersensitivity to stimuli, whereas its depletion strikingly reduces platelet reactivity. ${ }^{73}$ Moreover, HDL inhibit platelet fibrinogen binding and aggregation in response to thrombin via decreased formation of the second messengers diacylglycerol and inositol trisphosphate, ${ }^{74}$ and enhance platelet NO generation, thus increasing cyclic guanosine monophosphate, by acting on the apoER2 receptor. $^{75}$ Finally, HDL also downregulate the coagulation cascade and stimulate fibrinolysis. ${ }^{76}$

Considering the above summarized mechanisms, PCSK9 inhibition may modulate the effects of lipoproteins on platelets at various levels, thus reducing platelet activation. By strikingly decreasing plasma LDL-c, PCSK9 inhibitors may deplete platelet membranes of cholesterol, thus reducing platelet reactivity and pro-coagulant activity. In this regard, it is interesting that treatment of hypercholesterolaemic subjects with rosuvastatin, a powerful cholesterol-lowering statin, reduced platelet membrane cholesterol, TF expression and generation of FXa. ${ }^{77}$

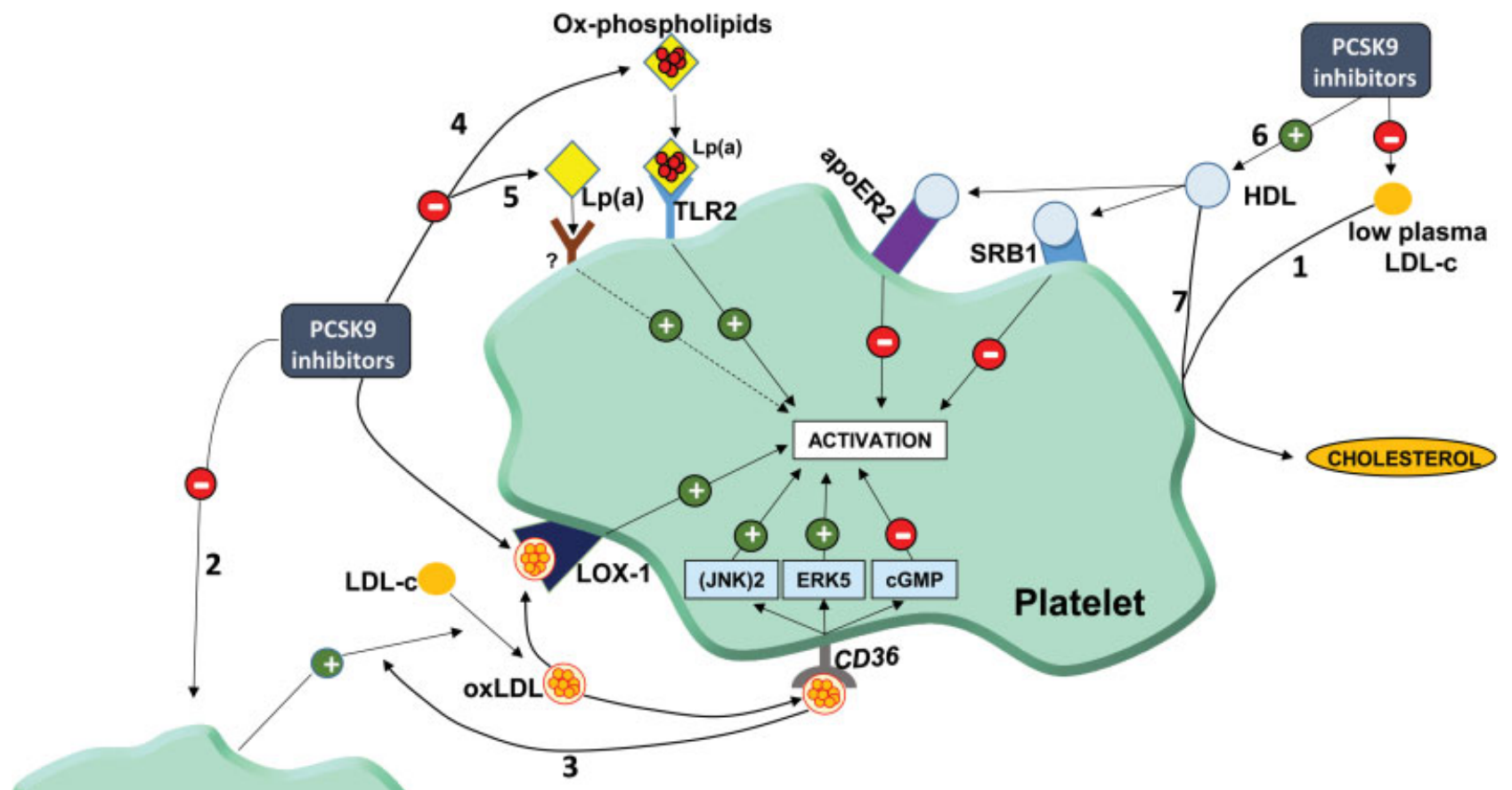

Activated platelet

Fig. 1 Hypothetical effects of proprotein convertase subtilisin/kexin type-9 (PCSK9) and its inhibition on platelets. (1) PCSK9 inhibition strikingly reduces low-density lipoprotein cholesterol (LDL-c) levels, thus potentially depleting platelet membranes of cholesterol, a mechanism reducing platelet reactivity. ${ }^{73}$ Moreover, the inhibition of platelet activation by PCSK9 inhibitors (2), a result of the various effects shown in the figure, may also reduce the ability of platelets to oxidize LDL thus decreasing the platelet stimulating activity of the latter through CD36 and LOX1 receptors (3). Furthermore, PCSK9 inhibition reduces lipoprotein (a) [LP(a)] levels, the main carriers of ox-phospholipids, thus potentially blunting their ability to activate platelets either through the Toll-like receptor 2 (TRL2) receptor (4) or directly (5). Finally, PCSK9 inhibition increases high-density lipoprotein (HDL) which reduces platelet activation acting on apoER2 and SRB1 receptors (6), and scavenging cholesterol from platelet membranes (7). +, stimulation; -, inhibition. 
In addition, given that PCSK9 and LOX-1 positively influence the expression of each other, ${ }^{78}$ it can be envisaged that PCSK9 inhibitors may reduce platelet LOX-1.

Moreover, the blunting of platelet activation by PCSK9 inhibitors may decrease ox-LDL generation, ${ }^{68}$ interrupting the vicious circle that propagates platelet activation. Indeed, treatment of hypercholesterolaemic patients with alirocumab or evolocumab was shown to reduce platelet activation. ${ }^{79}$ PCSK9 inhibitors, differently from statins, lower Lp(a) levels, ${ }^{26,80}$ and thus they may reduce its direct and oxidized phospholipid-mediated stimulatory effect on platelets. In addition, PCSK9 inhibitors also enhance HDL levels, ${ }^{25}$ in this way potentially inhibiting platelet aggregation directly or by depleting platelet membrane cholesterol ${ }^{72-75}$ (- Fig. 1).

Finally, cell apoptosis is known to favour thrombosis, in part through the release of pro-coagulant microparticles, ${ }^{81}$ and PCSK9 was found to enhance apoptosis in vascular smooth muscle and endothelial cells. ${ }^{82,83}$ In this context, PCSK9 inhibitors, by mitigating apoptosis, might indirectly prevent thrombosis.

\section{Regulation of FVIII Levels by LDLR and the Hypothetical Effect of PCSK9 Inhibitors}

Blood clotting FVIII is a key plasma protein, encoded by a gene located on chromosome $X$, which plays a central role in coagulation. FVIII acts as a co-factor for FIX, thus favouring thrombin generation. FVIII is transported in the circulation by von Willebrand factor (VWF) which stabilizes it and reduces its clearance. ${ }^{84}$
Epidemiologic studies have shown an association between increased FVIII plasma levels and arterial thrombosis, and elevated FVIII levels were found to correlate with a higher recurrence rate in patients with a prior myocardial infarction or ischaemic stroke. ${ }^{85-90}$ In contrast, haemophilia patients appear to be protected from ischaemic heart disease. ${ }^{91}$

Experimental studies in animal models, although often involving the use of supra-physiologic concentrations, provide further evidence in support of the role of FVIII in arterial thrombosis. ${ }^{36,92,93}$ The increased risk of arterial thrombosis associated with enhanced FVIII levels is thought to be due to the combination of increased thrombin generation and enhanced platelet adhesion/aggregation, the latter being induced by the concomitant increase of VWF. ${ }^{94,95}$

Circulating levels of FVIII are regulated by its biosynthesis and by its clearance through hepatic LDLR and lipoprotein receptor-related protein 1 (LRP1), both members of the LDLR family (-Fig. 2). FVIII is composed of a heavy and a light chain, the latter containing the binding site for LRP1, which is normally covered by VWF. ${ }^{96}$ Therefore, circulating FVIII not bound to VWF ( $\sim 5 \%$ of total) is quickly recognized by hepatocyte LRP1, endocytosed and degraded. ${ }^{97}$ The important role of LRP1 in FVIII clearance in vivo has been confirmed by the strong elevation of circulating FVIII levels in LRP-deficient mice as well as in mice with adenovirus-mediated over-expression of receptor-associated protein, a chaperone for LRP1 which blocks the binding of all ligands to the receptor. ${ }^{98}$ On the other hand, LDLR plays a role in FVIII clearance too because the simultaneous deletion of the LDLR and LRP1 genes in mice (double LRP1/ $\mathrm{LDLR}^{-1-}$ ) further enhanced FVIII levels by 4.2 -fold, while the

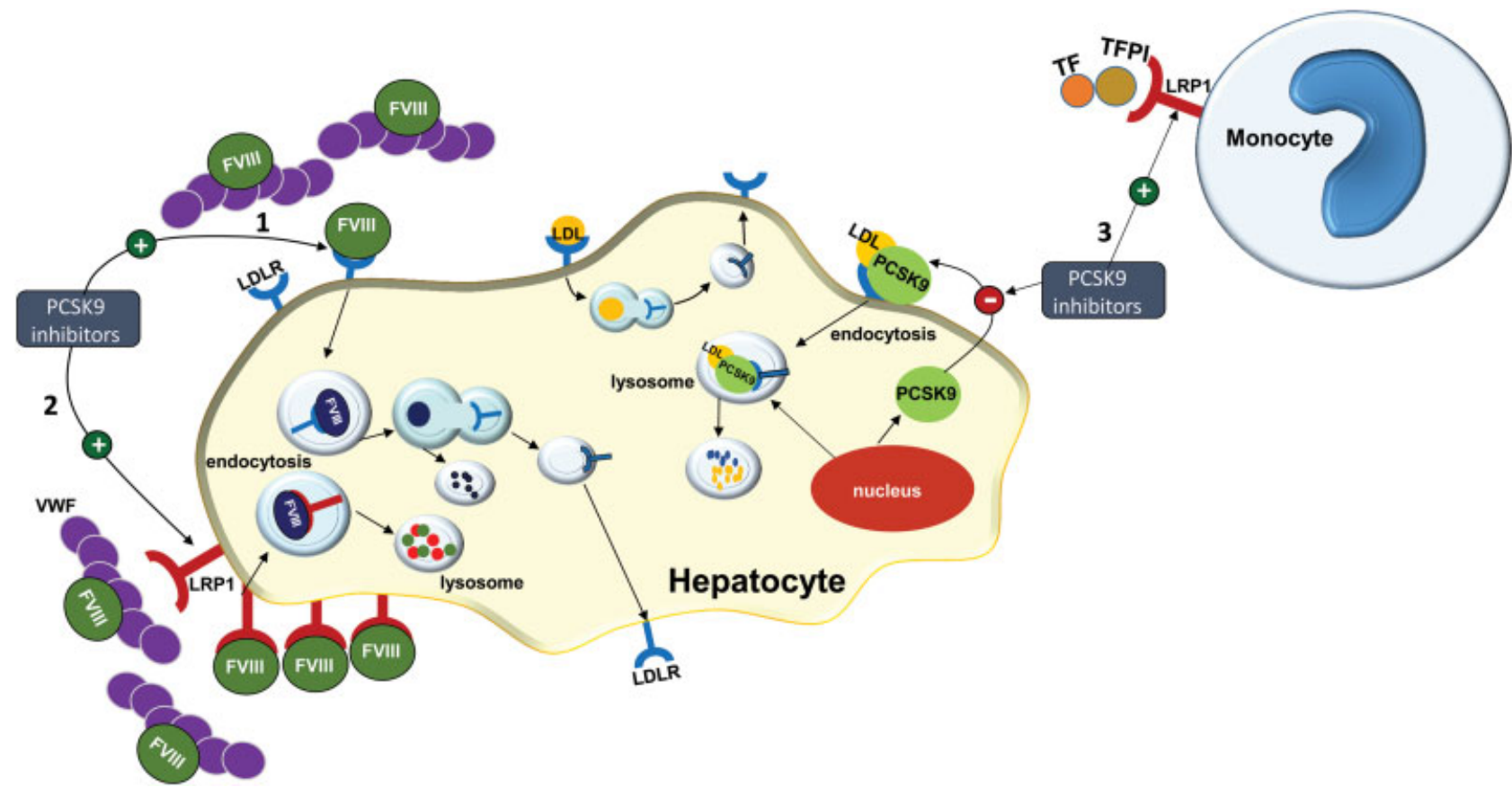

Fig. 2 Hypothetical effects of proprotein convertase subtilisin/kexin type-9 (PCSK9) inhibitors on factor VIII (FVIII), von Willebrand factor (VWF) and tissue factor levels. Hepatic low-density lipoprotein receptor (LDLR) and lipoprotein receptor-related protein 1 (LRP1) are both involved in the clearance of FVIII and VWF by the liver. PCSK9 inhibitors enhance the expression of hepatic LDLR, and possibly also of LRP-1(1), by both intraand extracellular mechanisms (2), thus potentially enhancing FVIII internalization and degradation leading to a decrease in FVIII plasma levels. Moreover, the possible enhancement of LRP1 by PCSK9 inhibitors in monocytes (3), might reduce circulating tissue factor (TF) through its accelerated clearance. +, stimulation; -, inhibition. 
Table 1 Hypothetical pleiotropic effects of PCSK9 inhibitors in atherothrombosis

\begin{tabular}{|l|l|l|l|}
\hline $\begin{array}{l}\text { Cell/tissue } \\
\text { targets }\end{array}$ & Reported PCSK9 effect & $\begin{array}{l}\text { Possible impact of PCSK9 } \\
\text { inhibition }\end{array}$ & Hypothetical mechanisms \\
\hline Platelets & $\begin{array}{l}\text { Platelet activation } \\
\text { and secretion } 32 \\
\text { Impaired response to } \\
\text { antiplatelet agents }\end{array}$ & $\begin{array}{l}\text { Platelet inhibition } \\
\text { Enhanced response to } \\
\text { antiplatelet agents }\end{array}$ & $\begin{array}{l}\text { I9hibition of ox-LDL, Lp(a)-mediated activation } \\
\text { Inhibition of CD36 and LOX1 receptor-mediated } \\
\text { activation } \\
\text { Enhancement of HDL-mediated inhibition }\end{array}$ \\
\hline $\begin{array}{l}\text { Blood } \\
\text { coagulation }\end{array}$ & No data & $\begin{array}{l}\text { Reduction of } \\
\text { circulating FVIII }\end{array}$ & $\begin{array}{l}\text { Enhanced LDLR and LRP1-mediated } \\
\text { FVIII clearance }\end{array}$ \\
\hline Tissue factor & $\begin{array}{l}\text { Positive correlation between } \\
\text { PCSK9 and TF plasma levels }\end{array}$ & $\begin{array}{l}\text { Reduced blood } \\
\text { clotting activation }\end{array}$ & $\begin{array}{l}\text { Enhanced monocyte LRP1- mediated } \\
\text { TF-TFPI complex clearance }\end{array}$ \\
\hline
\end{tabular}

Abbreviations: FVIII, factor VIII; HDL, high-density lipoprotein; LDL, low-density lipoprotein; LDLR, low-density lipoprotein receptor; Lp(a), lipoprotein (a); LRP1, lipoprotein receptor-related protein 1; TF, tissue factor; TFPI, tissue factor pathway inhibitor; PCSK9, proprotein convertase subtilisin/kexin type-9.

adenovirus-induced over-expression of hepatic LDLR accelerated the clearance of FVIII. ${ }^{99}$ These data show that LDLR cooperates with LRP1 in reducing FVIII levels, and indeed polymorphisms of the LDLR gene were found to affect CAD risk in humans by modulating FVIII:C levels and independently from the lipid profile. ${ }^{100}$ Monocyte LRP1 favours also the clearance of TF by mediating the internalization and degradation of the TF-TF pathway inhibitor complex. ${ }^{101}$

Currently, there are no data showing that FVIII levels may be influenced by PCSK9 inhibitors. However, it is conceivable that PCSK9 inhibitors, by strikingly increasing LDLR expression, may enhance the clearance of FVIII, thus reducing its plasma levels, a mechanism potentially contributing to the lowering of major adverse cardiovascular events (-Table $\mathbf{1}$ ). In this regard, it is relevant that data from the Multi-Ethnic Study of Atherosclerosis, a cohort study of healthy subjects free of clinical cardiovascular disease, showed that statin users had significantly lower FVIII levels. ${ }^{102}$ Moreover, a recent multi-centre, randomized, controlled, open-label study in patients with prior deep vein thrombosis, showed that a short-term course of high-dose rosuvastatin significantly reduced FVIII. ${ }^{103}$ Statins besides inhibiting of HMGCoA reductase, also increase $\operatorname{LDLR}^{104}$ and, at least atorvastatin, also LRP1. ${ }^{105}$ Therefore, FVIII reduction by statins is likely explained by the increased expression of LDLR and LRP1 and the resulting accelerated hepatic clearance of FVIII.

Although the hypothesis that PCSK9 inhibition may reduce FVIII levels by increasing its clearance seems plausible, experimental studies confirming it are required.

\section{Conclusion}

Several clues suggest that PCSK9 represents a major actor in cardiovascular disease, in part independently from its effects on lipid metabolism. Data from observational studies in humans and from experimental research in animals imply that PCSK9 may modulate both primary and secondary haemostasis either indirectly, through its effect on LDL-c, or directly by influencing platelet activation and plasma levels of FVIII. Previous observations, showing that the benefits of statins on cardiovascular events occur before any significant changes in lipid profile have taken place, ${ }^{19}$ have opened the way to a series of studies which unravelled several cholesterol-independent actions of this class of drugs, including the stabilization of atherosclerotic plaques, the improvement of endothelial function, the modulation of immune responses, the inhibition of oxidative stress and inflammation and the prevention of thrombosis. These studies not only have led to an advancement in our understanding of the role of inflammation in atherothrombosis, but also to the development of innovative therapeutic approaches targeting inflammation, like the anti-interleukin-1ß MoAb canakinumab. ${ }^{106}$ A complete unravelling of the possible pleiotropic activities of PCSK9 inhibitors, and in particular of their possible anti-thrombotic effects, may potentially widen the indications for this new therapeutic class and clarify their potential role in the treatment of the acute phase of ischaemic cardiovascular disease. The recent publication of the ODISSEY Trial shows that alirocumab reduced cardiovascular events and mortality in patients with a recent acute coronary syndrome and not on target for LDL-c on statin therapy, confirming that PCSK9 inhibitor therapy has an important role in secondary cardiovascular prevention in patients at high risk. ${ }^{107}$

\section{Funding}

This work was supported in part by a grant from Regione Umbria (Progetto di ricerca finalizzata - BANDO 2013) to P.G.

Conflict of Interest

None declared.

Acknowledgements

We thank Dr. A.S. Weyrich and Dr. R.A. Campbell (Department of Internal Medicine, Utah University, Salt Lake City, United States) for sharing unpublished data on megakaryocyte transcriptome.

\section{References}

1 Goldstein JL, Brown MS. Regulation of low-density lipoprotein receptors: implications for pathogenesis and therapy of hypercholesterolemia and atherosclerosis. Circulation 1987;76 (03):504-507 
2 Grundy SM, Stone NJ, Bailey AL, et al. AHA/ACC/AACVPR/AAPA/ ABC/ACPM/ADA/AGS/APhA/ASPC/NLA/PCNA Guideline on the management of blood cholesterol: a report of the American College of Cardiology/American Heart Association Task Force on clinical practice guidelines. J Am Coll Cardiol 2018. Doi: 10.1016/ j.jacc.2018.11.002

3 Santos-Gallego CG, Badimon JJ, Rosenson RS. Beginning to understand high-density lipoproteins. Endocrinol Metab Clin North Am 2014;43(04):913-947

4 Pedersen TR. The success story of LDL cholesterol lowering. Circ Res 2016;118(04):721-731

5 Segrest JP, Jones MK, De Loof H, Dashti N. Structure of apolipoprotein B-100 in low density lipoproteins. J Lipid Res 2001;42 (09):1346-1367

6 Brown MS, Anderson RG, Basu SK, Goldstein JL. Recycling of cellsurface receptors: observations from the LDL receptor system. Cold Spring Harb Symp Quant Biol 1982;46(Pt 2):713-721

7 Brown MS, Goldstein JL. A receptor-mediated pathway for cholesterol homeostasis. Science 1986;232(4746):34-47

8 Zhang DW, Garuti R, Tang WJ, Cohen JC, Hobbs HH. Structural requirements for PCSK9-mediated degradation of the low-density lipoprotein receptor. Proc Natl Acad Sci U S A 2008;105(35): 13045-13050

9 Abifadel M, Varret M, Rabès JP, et al. Mutations in PCSK9 cause autosomal dominant hypercholesterolemia. Nat Genet 2003;34 (02):154-156

10 Kent ST, Rosenson RS, Avery CL, et al. PCSK9 loss-of-function variants, low-density lipoprotein cholesterol, and risk of coronary heart disease and stroke: data from 9 studies of blacks and whites. Circ Cardiovasc Genet 2017;10(04):e001632

11 Maxwell KN, Breslow JL. Adenoviral-mediated expression of Pcsk9 in mice results in a low-density lipoprotein receptor knockout phenotype. Proc Natl Acad Sci U S A 2004;101(18):7100-7105

12 Lagace TA. PCSK9 and LDLR degradation: regulatory mechanisms in circulation and in cells. Curr Opin Lipidol 2014;25(05): 387-393

13 Sabatine MS, Giugliano RP, Keech AC, et al; FOURIER Steering Committee and Investigators. Evolocumab and clinical outcomes in patients with cardiovascular disease. N Engl J Med 2017;376 (18):1713-1722

14 Navarese EP, Kolodziejczak M, Schulze V, et al. Effects of proprotein convertase subtilisin/kexin type 9 antibodies in adults with hypercholesterolemia: a systematic review and meta-analysis. Ann Intern Med 2015;163(01):40-51

15 Ridker PM. Mortality differences associated with treatment responses in CANTOS and FOURIER: insight and implications. Circulation 2018;137(17):1763-1766

16 Liao JK, Laufs U. Pleiotropic effects of statins. Annu Rev Pharmacol Toxicol 2005;45:89-118

17 Ridker PM, Danielson E, Fonseca FA, et al; JUPITER Study Group. Rosuvastatin to prevent vascular events in men and women with elevated C-reactive protein. N Engl J Med 2008;359(21):2195-2207

18 Baigent C, Keech A, Kearney PM, et al; Cholesterol Treatment Trialists' (CTT) Collaborators. Efficacy and safety of cholesterollowering treatment: prospective meta-analysis of data from 90,056 participants in 14 randomised trials of statins. Lancet 2005;366(9493):1267-1278

19 Ludman A, Venugopal V, Yellon DM, Hausenloy DJ. Statins and cardioprotection-more than just lipid lowering? Pharmacol Ther 2009;122(01):30-43

20 Blum A, Shamburek R. The pleiotropic effects of statins on endothelial function, vascular inflammation, immunomodulation and thrombogenesis. Atherosclerosis 2009;203(02):325-330

21 Krysiak R, Okopień B, Herman Z. Effects of HMG-CoA reductase inhibitors on coagulation and fibrinolysis processes. Drugs 2003; 63(17):1821-1854

22 Antonopoulos AS, Margaritis M, Shirodaria C, Antoniades C. Translating the effects of statins: from redox regulation to suppression of vascular wall inflammation. Thromb Haemost 2012;108(05):840-848

23 Oesterle A, Laufs U, Liao JK. Pleiotropic effects of statins on the cardiovascular system. Circ Res 2017;120(01):229-243

24 Silverman MG, Ference BA, Im K, et al. Association between lowering LDL-C and cardiovascular risk reduction among different therapeutic interventions: a systematic review and metaanalysis. JAMA 2016;316(12):1289-1297

25 Filippatos TD, Kei A, Rizos CV, Elisaf MS. Effects of PCSK9 Inhibitors on other than low- density lipoprotein cholesterol lipid variables. J Cardiovasc Pharmacol Ther 2018;23(01): 3-12

26 Gaudet D, Kereiakes DJ, McKenney JM, et al. Effect of alirocumab, a monoclonal proprotein convertase subtilisin/kexin 9 antibody, on lipoprotein(a) concentrations (a pooled analysis of $150 \mathrm{mg}$ every two weeks dosing from phase 2 trials). Am J Cardiol 2014; 114(05):711-715

27 Bittner V. Pleiotropic effects of PCSK9 (proprotein convertase subtilisin/kexin type 9) inhibitors? Circulation 2016;134(22): 1695-1696

28 Santos-Gallego CG, Picatoste B, Badimón JJ. Pathophysiology of acute coronary syndrome. Curr Atheroscler Rep 2014;16(04): 401-409

29 Santos-Gallego CG, Bayón J, Badimón JJ. Thrombi of different pathologies: implications for diagnosis and treatment. Curr Treat Options Cardiovasc Med 2010;12(03):274-291

30 Gresele P, Momi S, Migliacci R. Endothelium, venous thromboembolism and ischaemic cardiovascular events. Thromb Haemost 2010;103(01):56-61

31 Migliacci R, Becattini C, Pesavento R, et al. Endothelial dysfunction in patients with spontaneous venous thromboembolism. Haematologica 2007;92(06):812-818

32 Camera M, Rossetti L, Barbieri SS, et al. PCSK9 as a positive modulator of platelet activation. J Am Coll Cardiol 2018;71(08): 952-954

33 Kassassir H, Siewiera K, Sychowski R, Watała C. Can the antiplatelet effects of cangrelor be reliably studied in mice under in vivo and in vitro conditions using flow cytometry? Pharmacol Rep 2013;65(04):870-883

34 Dwivedi DJ, Grin PM, Khan M, et al. Differential expression of PCSK9 modulates infection, inflammation, and coagulation in a murine model of sepsis. Shock 2016;46(06):672-680

35 Wang H, Wang Q Wang J, et al. Proprotein convertase subtilisin/ kexin type 9 (PCSK9) deficiency is protective against venous thrombosis in mice. Sci Rep 2017;7(01):14360

36 Emmerechts J, Vanassche T, Loyen S, et al. Partial versus complete factor VIII inhibition in a mouse model of venous thrombosis. Thromb Res 2012;129(04):514-519

37 Golder M, Mewburn J, Lillicrap D. In vitro and in vivo evaluation of the effect of elevated factor VIII on the thrombogenic process. Thromb Haemost 2013;109(01):53-60

38 Falcinelli E, Francisci D, Belfiori B, et al. In vivo platelet activation and platelet hyperreactivity in abacavir-treated HIV-infected patients. Thromb Haemost 2013;110(02):349-357

39 Navarese EP, Kolodziejczak M, Winter MP, et al. Association of PCSK9 with platelet reactivity in patients with acute coronary syndrome treated with prasugrel or ticagrelor: the PCSK9REACT study. Int J Cardiol 2017;227:644-649

40 Gresele P, Falcinelli E, Momi S. Potentiation and priming of platelet activation: a potential target for antiplatelet therapy. Trends Pharmacol Sci 2008;29(07):352-360

41 Rossetti L, Ferri N, Marchiano S, et al. PCSK9 beyond its role in cholesterol homeostasis: co-activator of platelet function. Eur Heart J 2017;38:446-447

42 Gresele P, Guglielmini G, De Angelis M, et al. Acute, short-term hyperglycemia enhances shear stress-induced platelet activation in patients with type II diabetes mellitus. J Am Coll Cardiol 2003;41(06):1013-1020 
43 Gresele P, Marzotti S, Guglielmini G, et al. Hyperglycemiainduced platelet activation in type 2 diabetes is resistant to aspirin but not to a nitric oxide-donating agent. Diabetes Care 2010;33(06):1262-1268

44 Rowley JW, Oler AJ, Tolley ND, et al. Genome-wide RNA-seq analysis of human and mouse platelet transcriptomes. Blood 2011;118(14):e101-e111

45 Cecchetti L, Tolley ND, Michetti N, Bury L, Weyrich AS, Gresele P. Megakaryocytes differentially sort mRNAs for matrix metalloproteinases and their inhibitors into platelets: a mechanism for regulating synthetic events. Blood 2011;118(07):1903-1911

46 Brambilla M, Facchinetti L, Canzano P, et al. Human megakaryocytes confer tissue factor to a subset of shed platelets to stimulate thrombin generation. Thromb Haemost 2015;114 (03):579-592

47 Radziwon-Balicka A, Lesyk G, Back V, et al. Differential eNOSsignalling by platelet subpopulations regulates adhesion and aggregation. Cardiovasc Res 2017;113(14):1719-1731

48 Li S, Zhu CG, Guo YL, et al. The relationship between the plasma PCSK9 levels and platelet indices in patients with stable coronary artery disease. J Atheroscler Thromb 2015;22(01):76-84

49 Cariou B, Guérin P, Le May C, et al. Circulating PCSK9 levels in acute coronary syndrome: results from the PC-SCA-9 prospective study. Diabetes Metab 2017;43(06):529-535

50 Fitzgerald DJ, Roy L, Catella F, FitzGerald GA. Platelet activation in unstable coronary disease. N Engl J Med 1986;315(16):983-989

51 Gresele P, Falcinelli E, Loffredo F, et al. Platelets release matrix metalloproteinase-2 in the coronary circulation of patients with acute coronary syndromes: possible role in sustained platelet activation. Eur Heart J 2011;32(03):316-325

52 De Servi S, Crimi G, Calabrò P, et al. Relationship between diabetes, platelet reactivity, and the SYNTAX score to one-year clinical outcome in patients with non-ST-segment elevation acute coronary syndrome undergoing percutaneous coronary intervention. EuroIntervention 2016;12(03):312-318

53 Hu D, Yang Y, Peng DQ. Increased sortilin and its independent effect on circulating proprotein convertase subtilisin/kexin type 9 (PCSK9) in statin-naive patients with coronary artery disease. Int J Cardiol 2017;227:61-65

54 Gustafsen C, Kjolby M, Nyegaard M, et al. The hypercholesterolemia-risk gene SORT1 facilitates PCSK9 secretion. Cell Metab 2014;19(02):310-318

55 Pastori D, Nocella C, Farcomeni A, et al; ATHERO-AF Study Group. Relationship of PCSK9 and urinary thromboxane excretion to cardiovascular events in patients with atrial fibrillation. J Am Coll Cardiol 2017;70(12):1455-1462

56 Catella F, FitzGerald GA. Paired analysis of urinary thromboxane B2 metabolites in humans. Thromb Res 1987;47(06): 647-656

57 Tatsumi K, Mackman N. Tissue factor and atherothrombosis. J Atheroscler Thromb 2015;22(06):543-549

58 Wang M, Li YF, Guo YG, Chen MM, Jiang ZL, Song JY. Positive correlation between plasma PCSK9 and tissue factors levels in patients with angiographically diagnosed coronary artery disease and diabetes mellitus. J Geriatr Cardiol 2016;13(04): 312-315

59 Ochoa E, Iriondo M, Manzano C, et al. LDLR and PCSK9 are associated with the presence of antiphospholipid antibodies and the development of thrombosis in aPLA carriers. PLoS One 2016;11(01):e0146990

60 Urbanus RT, de Laat B. Antiphospholipid antibodies and the protein C pathway. Lupus 2010;19(04):394-399

61 Bontadi A, Ruffatti A, Falcinelli E, et al. Platelet and endothelial activation in catastrophic and quiescent antiphospholipid syndrome. Thromb Haemost 2013;109(05):901-908

62 Obermayer G, Afonyushkin T, Binder CJ. Oxidized low-density lipoprotein in inflammation-driven thrombosis. J Thromb Haemost 2018;16(03):418-428
63 Chen K, Febbraio M, Li W, Silverstein RL. A specific CD36dependent signaling pathway is required for platelet activation by oxidized low-density lipoprotein. Circ Res 2008;102(12): 1512-1519

64 Hofmann A, Brunssen C, Morawietz H. Contribution of lectin-like oxidized low-density lipoprotein receptor-1 and LOX-1 modulating compounds to vascular diseases. Vascul Pharmacol 2017. Doi: 10.1016/j.vph.2017.10.002

65 Magwenzi S, Woodward C, Wraith KS, et al. Oxidized LDL activates blood platelets through CD36/NOX2-mediated inhibition of the CGMP/protein kinase G signaling cascade. Blood 2015; 125(17):2693-2703

66 Yoshimoto R, Fujita Y, Kakino A, Iwamoto S, Takaya T, Sawamura T. The discovery of LOX-1, its ligands and clinical significance. Cardiovasc Drugs Ther 2011;25(05):379-391

67 Marwali MR, Hu CP, Mohandas B, et al. Modulation of ADPinduced platelet activation by aspirin and pravastatin: role of lectin-like oxidized low-density lipoprotein receptor-1, nitric oxide, oxidative stress, and inside-out integrin signaling. J Pharmacol Exp Ther 2007;322(03):1324-1332

68 Carnevale R, Bartimoccia S, Nocella C, et al. LDL oxidation by platelets propagates platelet activation via an oxidative stressmediated mechanism. Atherosclerosis 2014;237(01):108-116

69 Biswas S, Xin L, Panigrahi S, et al. Novel phosphatidylethanolamine derivatives accumulate in circulation in hyperlipidemic ApoE-/-mice and activate platelets via TLR2. Blood 2016;127(21):2618-2629

70 Leibundgut G, Scipione C, Yin H, et al. Determinants of binding of oxidized phospholipids on apolipoprotein (a) and lipoprotein (a). J Lipid Res 2013;54(10):2815-2830

71 Riches K, Porter KE. Lipoprotein(a): cellular effects and molecular mechanisms. Cholesterol 2012;2012:923289

72 Calkin AC, Drew BG, Ono A, et al. Reconstituted high-density lipoprotein attenuates platelet function in individuals with type 2 diabetes mellitus by promoting cholesterol efflux. Circulation 2009;120(21):2095-2104

73 Shattil SJ, Anaya-Galindo R, Bennett J, Colman RW, Cooper RA. Platelet hypersensitivity induced by cholesterol incorporation. J Clin Invest 1975;55(03):636-643

74 Nofer JR, Walter M, Kehrel B, et al. HDL3-mediated inhibition of thrombin-induced platelet aggregation and fibrinogen binding occurs via decreased production of phosphoinositide-derived second messengers 1,2-diacylglycerol and inositol 1,4,5-tris-phosphate. Arterioscler Thromb Vasc Biol 1998;18(06):861-869

75 Riddell DR, Graham A, Owen JS. Apolipoprotein E inhibits platelet aggregation through the L-arginine:nitric oxide pathway. Implications for vascular disease. J Biol Chem 1997;272(01):89-95

76 van der Stoep M, Korporaal SJ, Van Eck M. High-density lipoprotein as a modulator of platelet and coagulation responses. Cardiovasc Res 2014;103(03):362-371

77 Panes O, González C, Hidalgo P, et al. Platelet tissue factor activity and membrane cholesterol are increased in hypercholesterolemia and normalized by rosuvastatin, but not by atorvastatin. Atherosclerosis 2017;257:164-171

78 Ding Z, Liu S, Wang X, et al. Cross-talk between LOX-1 and PCSK9 in vascular tissues. Cardiovasc Res 2015;107(04):556-567

79 Barale C, Bonomo K, Noto F, et al. Effects of PCSK9 inhibitors on platelet function in adults with hypercholesterolemia. Atherosclerosis 2017;263:e30-e31

80 Kotani K, Banach M. Lipoprotein(a) and inhibitors of proprotein convertase subtilisin/kexin type 9. J Thorac Dis 2017;9(01):E78-E82

81 Geddings JE, Mackman N. New players in haemostasis and thrombosis. Thromb Haemost 2014;111(04):570-574

82 Ding Z, Liu S, Wang X, et al. Cross-talk between PCSK9 and damaged mtDNA in vascular smooth muscle cells: role in apoptosis. Antioxid Redox Signal 2016;25(18):997-1008

83 Li J, Liang X, Wang Y, Xu Z, Li G. Investigation of highly expressed PCSK9 in atherosclerotic plaques and ox-LDL-induced endothelial cell apoptosis. Mol Med Rep 2017;16(02):1817-1825 
84 Kaufman RJ, Pipe SW, Tagliavacca L, Swaroop M, Moussalli M. Biosynthesis, assembly and secretion of coagulation factor VIII. Blood Coagul Fibrinolysis 1997;8(02, Suppl 2):S3-S14

85 Bank I, Libourel EJ, Middeldorp S, et al. Elevated levels of FVIII:C within families are associated with an increased risk for venous and arterial thrombosis. J Thromb Haemost 2005;3 (01):79-84

86 Siegler JE, Samai A, Albright KC, Boehme AK, Martin-Schild S. Factoring in factor VIII with acute ischemic stroke. Clin Appl Thromb Hemost 2015;21(07):597-602

87 Rumley A, Lowe GD, Sweetnam PM, Yarnell JW, Ford RP. Factor VIII, von Willebrand factor and the risk of major ischaemic heart disease in the Caerphilly Heart Study. Br J Haematol 1999;105 (01):110-116

88 Tracy RP, Arnold AM, Ettinger W, Fried L, Meilahn E, Savage P. The relationship of fibrinogen and factors VII and VIII to incident cardiovascular disease and death in the elderly: results from the cardiovascular health study. Arterioscler Thromb Vasc Biol 1999; 19(07):1776-1783

89 Folsom AR, Peacock JM, Boerwinkle E; Atherosclerosis Risk in Communities (ARIC) Study Investigators. Variation in PCSK9, low LDL cholesterol, and risk of peripheral arterial disease. Atherosclerosis 2009;202(01):211-215

90 Milgrom A, Lee K, Rothschild M, et al. Thrombophilia in 153 patients with premature cardiovascular disease $\leq$ age 45 . Clin Appl Thromb Hemost 2018;24(02):295-302

91 Rosendaal FR, Briët E, Stibbe J, et al. Haemophilia protects against ischaemic heart disease: a study of risk factors. Br J Haematol 1990;75(04):525-530

92 Machlus KR, Lin FC, Wolberg AS. Procoagulant activity induced by vascular injury determines contribution of elevated factor VIII to thrombosis and thrombus stability in mice. Blood 2011;118 (14):3960-3968

93 Kawasaki T, Kaida T, Arnout J, Vermylen J, Hoylaerts MF. A new animal model of thrombophilia confirms that high plasma factor VIII levels are thrombogenic. Thromb Haemost 1999;81(02):306-311

94 Brummel-Ziedins K, Undas A, Orfeo T, et al. Thrombin generation in acute coronary syndrome and stable coronary artery disease: dependence on plasma factor composition. J Thromb Haemost 2008;6(01):104-110

95 Rice GI, Grant PJ. FVIII coagulant activity and antigen in subjects with ischaemic heart disease. Thromb Haemost 1998;80(05): 757-762
96 Lenting PJ, Neels JG, van den Berg BM, et al. The light chain of factor VIII comprises a binding site for low density lipoprotein receptorrelated protein. J Biol Chem 1999;274(34):23734-23739

97 Saenko EL, Yakhyaev AV, Mikhailenko I, Strickland DK, Sarafanov AG. Role of the low density lipoprotein-related protein receptor in mediation of factor VIII catabolism. J Biol Chem 1999;274(53): 37685-37692

98 Bovenschen N, Herz J, Grimbergen JM, et al. Elevated plasma factor VIII in a mouse model of low-density lipoprotein receptorrelated protein deficiency. Blood 2003;101(10):3933-3939

99 Bovenschen N, Mertens K, Hu L, Havekes LM, van Vlijmen BJ. LDL receptor cooperates with LDL receptor-related protein in regulating plasma levels of coagulation factor VIII in vivo. Blood 2005;106(03):906-912

100 Martinelli N, Girelli D, Lunghi B, et al. Polymorphisms at LDLR locus may be associated with coronary artery disease through modulation of coagulation factor VIII activity and independently from lipid profile. Blood 2010;116(25):5688-5697

101 Hamik A, Setiadi H, Bu G, McEver RP, Morrissey JH. Downregulation of monocyte tissue factor mediated by tissue factor pathway inhibitor and the low density lipoprotein receptorrelated protein. J Biol Chem 1999;274(08):4962-4969

102 Adams NB, Lutsey PL, Folsom AR, et al. Statin therapy and levels of hemostatic factors in a healthy population: the Multi-Ethnic Study of Atherosclerosis. J Thromb Haemost 2013;11(06):1078-1084

103 Biedermann JS, Kruip MJHA, van der Meer FJ, et al. Rosuvastatin use improves measures of coagulation in patients with venous thrombosis. Eur Heart J 2018;39(19):1740-1747

104 Tiwari V, Khokhar M. Mechanism of action of anti-hypercholesterolemia drugs and their resistance. Eur J Pharmacol 2014; 741:156-170

105 Moon JH, Kang SB, Park JS, et al. Up-regulation of hepatic lowdensity lipoprotein receptor-related protein 1: a possible novel mechanism of antiatherogenic activity of hydroxymethylglutaryl-coenzyme A reductase inhibitor Atorvastatin and hepatic LRP1 expression. Metabolism 2011;60(07):930-940

106 Ridker PM, Everett BM, Thuren T, et al; CANTOS Trial Group. Antiinflammatory therapy with canakinumab for atherosclerotic disease; CANTOS Trial Group. N Engl J Med 2017;377(12): $1119-1131$

107 Schwartz GG, Steg PG, Szarek M, et al. Alirocumab and cardiovascular outcomes after acute coronary syndrome. N Engl J Med 2018;379:2097-2107 